\title{
A viabilidade do uso do pêndulo simples como ferramenta no ensino de física do ensino médio para o cálculo da aceleração da gravidade local
}

\author{
The feasibility of using the simple pendulum as a tool in teaching high school \\ physics to calculate the acceleration of local gravity
}

\begin{abstract}
Cristiano Rocha da Cunha '; Lúcio Ângelo Vidal "; Guilherme Tales da Silva "ll; Ruth Silva Pereira Iv; Rita Santana Ramos da Silva v; Willam Campos Tokashiki vi
\end{abstract}

\begin{abstract}
RESUMO
Conhecer o valor g da aceleração da gravidade é de suma importância em diversas análises, e há várias maneiras de obtê-lo experimentalmente. Aqui, buscando recursos de fácil aplicação em salas de aula do ensino médio, os autores optaram pela observação de um pêndulo simples, realizando uma série de medições do período do pêndulo e aplicando-as às equações (adequadamente manipuladas para este experimento) deste movimento oscilatório. O experimento foi realizado em quatro etapas: uma com uma massa de 10 gramas e três com uma massa de 20 gramas; duas com dez oscilações, uma com quinze e uma com vinte. Como resultado, foram estimados quatro valores da aceleração local, os quais foram validados, utilizando-se a teoria dos erros, com o valor disponibilizado na literatura. Com esses dados, verificou-se a viabilidade do uso deste dispositivo no processo de ensino-aprendizagem, dados a sua facilidade em manuseio e montagem, o seu baixo custo e o seu irrisório erro com o valor da literatura.
\end{abstract}

Palavras-chave: Pêndulo simples; Aceleração da gravidade; Ensino

\section{ABSTRACT}

Knowing the g-value of acceleration of gravity is of paramount importance in various analyzes, and there are several ways of obtaining it experimentally. Here, searching for resources that are easy to apply in high school classrooms, the authors opted for the observation of a simple pendulum, performing a series of measurements of the pendulum period and applying them to the equations (appropriately manipulated for this experiment) of this oscillatory movement. The experiment was carried out in four steps: one with a mass of 10 grams and three with a mass of 20 grams; two with ten swings, one with fifteen and one with twenty. As a result, four values of local acceleration were estimated, which were compared, using the theory of errors, with the value made available in the literature. With these data, the feasibility of using this device in the teaching-learning process was verified, given its ease of handling and assembly, its low cost and its negligible error with the value of the literature.

Keywords: Simple pendulum; Acceleration of gravity; Teaching 


\section{Introdução}

A investigação científica sobre o ensino de física na América Latina e no Brasil passou a ser difundida por volta da década de 1960, isso após uma implementação idêntica ter dado o pontapé inicial nos Estados Unidos, conforme nos lembram Werner da Rosa e Becker da Rosa (2012). Foi um momento em que houve um entusiasmo com o desenvolvimento da ciência e, de certa forma, a disciplina de física tinha a intenção de uma iniciação dos jovens às carreiras científicas.

Diante disso, nos dias atuais, o desafio dessa disciplina se tornou ainda maior por conta do aumento dos conteúdos das disciplinas do ensino médio. A tecnologia se tornou uma ferramenta para a melhoria no processo de ensino-aprendizagem e, sobretudo nas ciências, permitiu ao discente uma maior possibilidade de visualização da aplicação que se pode fazer da física com muitas outras áreas, como na medicina, na engenharia, na geotecnologia etc.

No entanto, quando se reporta ao ensino de física nas escolas públicas, o professor se depara com grandes dificuldades, como poucas horas de encontro com os alunos ao longo da semana, falta de laboratório de ensino, material didático precário, dentre outras.

No contexto de tantas dificuldades para uma aprendizagem significativa e de alunos com cada vez maior acesso à informação, surge uma necessidade de práticas que auxiliem no processo de ensino-aprendizagem do aluno, estimulem o interesse pela ciência e, sobretudo, sejam de baixo custo, dada a limitação orçamentária que as escolas públicas brasileiras enfrentam atualmente.

Dessa forma, este trabalho teve como objetivo geral a investigação de um método alternativo do uso do pêndulo simples para a determinação do valor local da aceleração da gravidade com o uso de materiais de baixo custo e de fácil obtenção e operação. Já os objetivos específicos do trabalho são: validar os valores encontrados com aqueles apresentados na literatura, apresentar os conceitos de frequência e período associados ao pêndulo simples e validar as equações da frequência e do período apresentadas pela literatura para este tipo de movimento harmônico simples.

\section{Referencial teórico}

\subsection{Movimento periódico}

Como definido por Young e Freedman (2008), é periódico qualquer movimento que se repete indefinidamente. Quando um corpo executa esse movimento, há uma posição de equilíbrio para onde esse corpo sempre retorna em razão de uma força (ou torque) restauradora. Para se descrever o movimento, existem quatro medidas fundamentais: a amplitude $(A)$, valor máximo do deslocamento do corpo em relação à posição de equilíbrio; o período $(T)$, intervalo de tempo decorrido durante um ciclo completo; a frequência $(f)$, número de oscilações por intervalo de tempo; e a frequência angular $(\omega)$, igual a $2 \pi f$, taxa de variação de uma grandeza angular ${ }^{1}$. No SI, as unidades dessas grandezas são, respectivamente, o metro $(\mathrm{m})$, o segundo $(\mathrm{s})$, o Hertz $\left(\mathrm{Hz}=\operatorname{ciclo} / \mathrm{s}=\mathrm{s}^{-1}\right)$ e o radiano por segundo $\left(=\operatorname{rad} / \mathrm{s}=\mathrm{s}^{-1}\right)$. O período $T$ e a frequência $f$ podem ser expressos da seguinte forma:

$$
T=\frac{\text { intervalo de tempo }}{\mathrm{n}^{\mathrm{o}} \text { de oscilações }} ; \quad f=\frac{\mathrm{n}^{\mathrm{o}} \text { de oscilações }}{\text { intervalo de tempo }}
$$

Assim, das definições dadas, verificam-se as seguintes relações:

$$
\begin{gathered}
f=\frac{1}{T} ; \quad T=\frac{1}{f} \\
\omega=2 \pi f=\frac{2 \pi}{T}
\end{gathered}
$$

\subsection{Movimento harmônico simples}

\subsubsection{Definição}

O movimento harmônico simples, ou MHS, é o tipo mais simples de movimento periódico. Em um sistema massa-mola, ele "ocorre quando a força restauradora $F_{x}$ é diretamente proporcional ao deslocamento $x$ da posição de equilíbrio" (Young e Freedman, 2008, p. 38). Matematicamente,

$$
F_{x}=-k x
$$

\footnotetext{
${ }^{1}$ A frequência angular relaciona o movimento periódico ao movimento circular. Ela indica a "velocidade", em radianos por segundo (relação com o círculo de referência), com a qual o corpo realiza o movimento periódico.
} 
onde $k$ é a constante da força. Em particular, num sistema massa-mola, $k$ é a constante elástica da mola. Portanto, a aceleração instantânea $a_{x}=F_{x} / m$ de um corpo de massa $m$ que efetua um MHS é dada por

$$
a_{x}=-\frac{k}{m} x
$$

\subsubsection{O MHS e o movimento circular uniforme}

É possível relacionar o MHS ao movimento circular uniforme (MCU) e assim deduzir as equações de posição, de velocidade e de aceleração do MHS. Aqui, apenas será mostrada essa relação e como a frequência angular $\omega$ aparece nela.

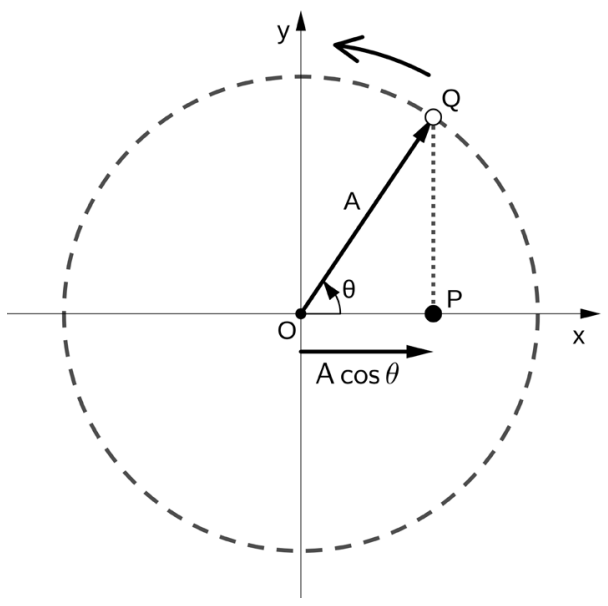

Figura 1: Posição no MHS como projeção de um MCU

Na Figura 1, o ponto $Q$ descreve um movimento circular uniforme de raio $A$ em torno da origem $O$, o ponto $P$ é a projeção de $Q$ sobre o eixo $O x$, e $\overrightarrow{O Q}$, um vetor girante chamado fasor, faz um ângulo $\theta$ com o eixo $+O x$. O ponto $Q$ e o fasor giram com velocidade angular constante $\omega$. No instante $t$ da Figura 1, a componente do fasor em $O x$ é a abscissa do ponto $Q$ e, portanto, corresponde à distância de $P$ à origem. Ou seja, sendo $x$ o deslocamento de $P$ da origem, tem-se

$$
x=A \cos \theta
$$

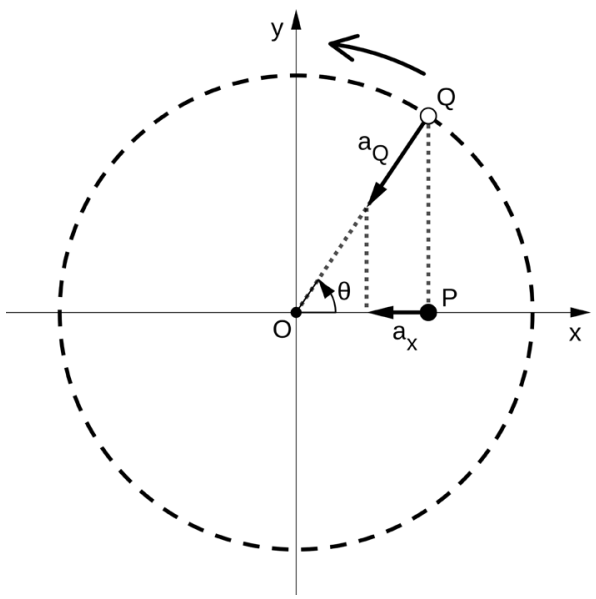

Figura 2: Aceleração no MHS como projeção de um MCU

Num movimento circular uniforme, o módulo da aceleração centrípeta é dada por

$$
a_{c}=\frac{\text { velocidade }^{2}}{\text { raio }}=\frac{\text { velocidade }^{2}}{\text { raio }^{2}} \times \text { raio }
$$




$$
\Rightarrow a_{c}=(\text { velocidade angular })^{2} \times \text { raio }
$$

Na Figura 2, $\overrightarrow{a_{Q}}$ é a aceleração centrípeta do ponto $Q$ e $\overrightarrow{a_{x}}$ é a componente de $\overrightarrow{a_{Q}}$ em $O x$. Assim, de acordo com a equação (5), pode-se escrever $a_{Q}=\omega^{2} A$. Portanto,

$$
a_{x}=-a_{Q} \cos \theta=-\omega^{2} A \cos \theta
$$

e, com base na equação (4),

$$
a_{x}=-\omega^{2} x
$$

A equação (6) tem exatamente as mesmas características da aceleração do movimento harmônico simples expressa na equação (3), desde que $\omega^{2}=k / m$, ou seja,

$$
\omega=\sqrt{\frac{k}{m}}
$$

Substituindo a equação (7) na equação (1) e isolando $f$ e $T$, obtém-se

$$
f=\frac{1}{2 \pi} \sqrt{\frac{k}{m}} ; \quad T=2 \pi \sqrt{\frac{m}{k}}
$$

\subsection{Pêndulo Simples}

\subsubsection{Definições e equações}

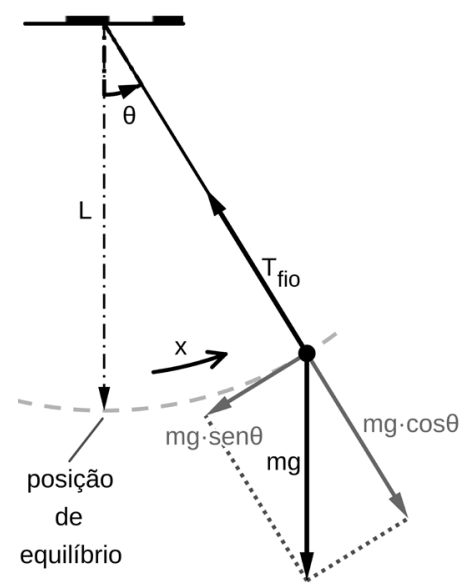

Figura 3: Pêndulo simples

Um pêndulo simples, objeto do experimento deste artigo, é "composto por uma partícula de massa $m$ (chamada de peso do pêndulo) suspensa por uma das extremidades de um fio inextensível, de massa desprezível e comprimento $L$, cuja outra extremidade está fixa" (Halliday et al., 2009, p. 95-96). A partícula oscila num plano e sobre ela atuam a força peso $m g$ e a tensão $T_{\text {fio }}$ do fio. Na Figura $3, \theta$ é o ângulo entre o fio e a vertical. O deslocamento $x$ do peso é a distância medida, a partir da posição de equilíbrio, ao longo do arco de circunferência de raio $L$. A força peso foi decomposta radial e tangencialmente à trajetória. Nesse sistema, a componente radial da força peso e a tensão do fio se anulam, pois elas têm a mesma direção e a mesma intensidade, porém sentidos opostos, e não há aceleração resultante na direção do fio. Desta forma, resta como resultante (e força restauradora do movimento) a componente tangencial

$$
F=-m g \operatorname{sen} \theta
$$

Para que o pêndulo em questão descrevesse um movimento harmônico simples, seria necessário que a força restauradora fosse diretamente proporcional ao deslocamento $x$ (e também ao ângulo $\theta$, pois $x=L \theta$ ). Porém, de acordo com a equação (9), isso não ocorre. Entretanto, para valores suficientemente pequenos de $\theta\left(\leqslant 10^{\circ}\right)$, tem-se $\operatorname{sen} \theta \approx \theta$. Admitindo-se então pequenos deslocamentos, pode-se escrever

$$
F=-m g \operatorname{sen} \theta \approx-m g \theta=-m g \frac{x}{L}
$$


ou seja,

$$
F=-\frac{m g}{L} x
$$

Relacionando as equações (10) e (2), obtém-se $k=m g / L$. Substituindo nas equações (7) e (8):

$$
\omega=\sqrt{\frac{g}{L}}, \quad f=\frac{1}{2 \pi} \sqrt{\frac{g}{L}}
$$

e

$$
T=2 \pi \sqrt{\frac{L}{g}}
$$

\subsubsection{Medindo a aceleração da gravidade}

Como Galileu constatou, a equação (11) mostra "que se pode relacionar o período de oscilação com o comprimento do fio que suspende o corpo oscilante do pêndulo" (Fuke e Yamamoto, 2010, p. 251), e juntamente com o valor g da aceleração da gravidade, pode-se calcular T. Mas nada impede que se faça o caminho contrário: sabendo o comprimento $L$ do fio e o período $T$ do pêndulo, encontrar a aceleração da gravidade. Isolando $g$ na equação (11), obtém-se

$$
g=\frac{4 \pi^{2} L}{T^{2}}
$$

Portanto, obtendo valores de $L$ e de $T$ por medições suficientemente precisas, basta substituí-los na equação (12) para encontrar uma boa aproximação de $g$.

\section{Materiais e métodos}

\subsection{Materiais e preparação do pêndulo}

Para a preparação do pêndulo, foram utilizados um tripé universal, uma linha fina de costura, dois pesos de $10 \mathrm{~g}$ cada e um pequeno suporte metálico para os pesos; e para as medições, uma fita métrica (trena) de $2 \mathrm{~m}$ e um cronômetro. (Ver Figura 4(a).) Na extremidade do tripé (Figura 4(b)), foi amarrado um pedaço da linha de costura, em cuja extremidade foi fixado o suporte metálico para os pesos.

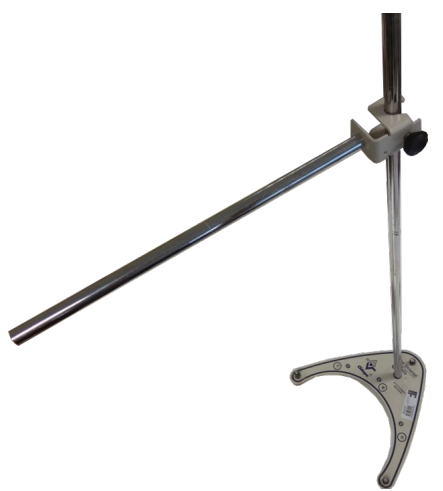

(a) Materiais

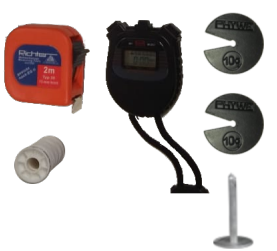

Figura 4: Preparação do pêndulo

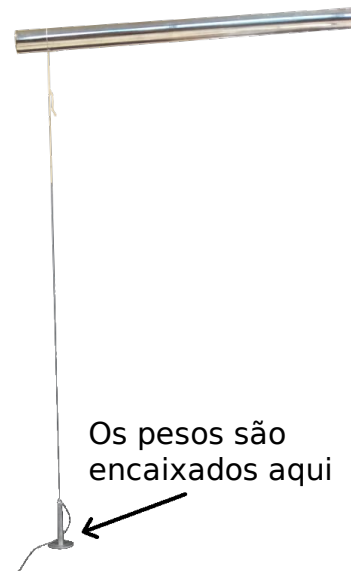

(b) Extremidade do tripé 


\subsection{Metodologia}

Antes de iniciar o experimento, com o auxílio da trena foi medido o comprimento $L$ do fio (Figura 5 ), obtendo-se aproximadamente $30 \mathrm{~cm}$.

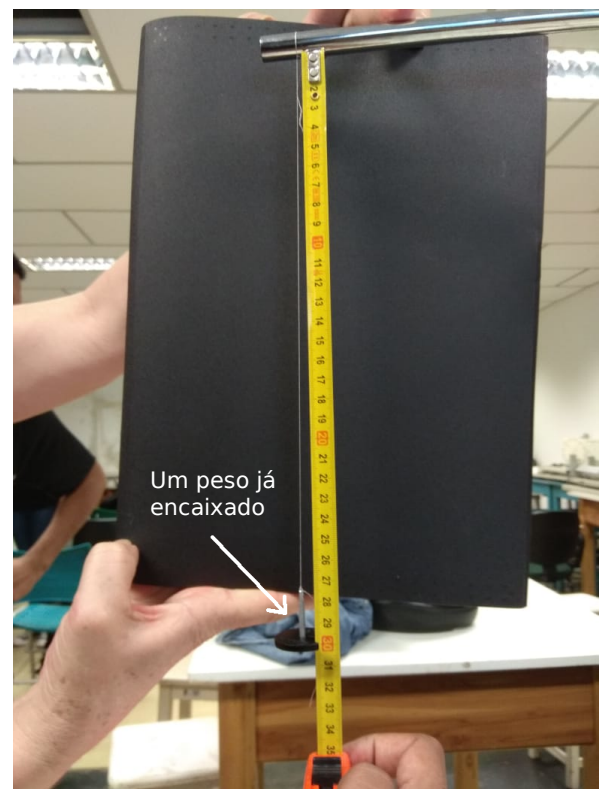

Figura 5: Medindo o comprimento do fio

O experimento consiste nos seguintes passos:

Passo 1 Colocar o(s) peso(s) no suporte metálico.

Passo 2 Puxar levemente o pêndulo da posição de equilíbrio, procurando não ultrapassar $10^{\circ}$ de inclinação em relação à vertical.

Passo 3 Com o cronômetro em mãos, zerado, acioná-lo e soltar o pêndulo simultaneamente.

Passo 4 Após uma quantidade inteira $n$ de oscilações, parar o cronômetro e anotar o tempo.

Passo 5 Repetir os passos 2, 3 e 4 várias vezes e fazer uma média dos tempos anotados no passo anterior.

Passo 6 Dividir por $n$ a média obtida para encontrar uma média do período $T$ do pêndulo.

Passo 7 Aplicar $L$ (medido inicialmente) e $T$ na equação (12) e assim obter uma aproximação de $g$.

Galileu Galilei (1564-1642) observou que, desprezando-se a resistência do ar, "objetos de vários pesos, soltos ao mesmo tempo, caíam juntos e atingiam o chão ao mesmo tempo" (Hewitt, 2011, p. 21). Sabemos também que "o período é independente da massa da partícula suspensa" (Halliday et al., 2013, p. 95) no fio (este movimento e a queda livre são ambos provocados pela ação da gravidade). Somente o comprimento do fio e a aceleração da gravidade influem no tempo de oscilação do pêndulo (equação (11)). Para verificar isso experimentalmente, foram utilizadas massas distintas na extremidade do pêndulo, dando-se melhor ênfase à maior massa em razão desta ser menos sujeita à ação das forças dissipativas (como a resistência do ar). Como o intervalo de tempo observado e a amplitude são pequenos, o sistema foi considerado conservativo, no qual "as forças que realizam trabalho transformam exclusivamente energia potencial em energia cinética e vice-versa" (Doca et al., 2013, p. 232).

Para um maior rigor nas medições, o experimento foi realizado em quatro etapas:

1. dez medições, um peso de $10 \mathrm{~g}$ na extremidade do fio e dez oscilações por medição;

2. dez medições, dois pesos de 10 g (massa total de 20 g) e dez oscilações por medição;

3. quatro medições, massa de $20 \mathrm{~g}$ (dois pesos) e quinze oscilações por medição;

4. cinco medições, massa de 20 g (dois pesos) e vinte oscilações por medição. 


\subsection{Dados de localização}

O experimento foi realizado no Laboratório de Física do IFMT Campus Cuiabá - Cel. Octayde Jorge da Silva -, que está aproximadamente na latitude de $15^{\circ}$ e a uma altitude de $221 \mathrm{~m}$ acima do nível do mar. (Dados obtidos com o aplicativo Google Earth.)

\section{Resultados e discussões}

Cada etapa do experimento retornou um valor diferente da aceleração da gravidade local, totalizando quatro resultados, os quais foram denotados, respectivamente, por $g_{1}, g_{2}, g_{3}$ e $g_{4}$.

\subsection{Etapa 1}

\begin{tabular}{cc} 
Tabela 1: Dados obtidos na $1^{\mathrm{a}}$ etapa \\
\hline$m=10 \mathrm{~g}, L=0,3 \mathrm{~m}, n=10$ oscilações \\
\hline \hline Medição $(i)$ & Tempo $\left(\Delta t_{i}\right)$ \\
\hline 1 & $10,82 \mathrm{~s}$ \\
2 & $10,85 \mathrm{~s}$ \\
3 & $10,88 \mathrm{~s}$ \\
4 & $10,81 \mathrm{~s}$ \\
5 & $10,87 \mathrm{~s}$ \\
6 & $10,90 \mathrm{~s}$ \\
7 & $10,75 \mathrm{~s}$ \\
8 & $10,81 \mathrm{~s}$ \\
9 & $10,90 \mathrm{~s}$ \\
10 & $10,90 \mathrm{~s}$ \\
\hline Média $(\Delta t)$ & $\sum \Delta t_{i} / 10=10,849 \mathrm{~s}$ \\
\hline
\end{tabular}

Calculando $T$ com os dados da Tabela 1 e aplicando-o com $L$ na equação (12), obteve-se

$$
T=\frac{\Delta t}{n}=\frac{10,849 \mathrm{~s}}{10}=1,0849 \mathrm{~s} \Rightarrow g_{1}=\frac{4 \pi^{2} \times(0,3 \mathrm{~m})}{(1,0849 \mathrm{~s})^{2}} \approx 10,0624 \mathrm{~m} / \mathrm{s}^{2}
$$

\subsection{Etapa 2}

Tabela 2: Dados obtidos na $2^{\mathrm{a}}$ etapa

\begin{tabular}{cc}
\hline$m=20 \mathrm{~g}, L=0,3 \mathrm{~m}, n=10$ oscilações \\
\hline \hline Medição $(i)$ & Tempo $\left(\Delta t_{i}\right)$ \\
\hline 1 & $10,91 \mathrm{~s}$ \\
2 & $10,88 \mathrm{~s}$ \\
3 & $10,81 \mathrm{~s}$ \\
4 & $10,94 \mathrm{~s}$ \\
5 & $10,75 \mathrm{~s}$ \\
6 & $10,84 \mathrm{~s}$ \\
7 & $10,84 \mathrm{~s}$ \\
8 & $10,90 \mathrm{~s}$ \\
9 & $10,88 \mathrm{~s}$ \\
10 & $10,90 \mathrm{~s}$ \\
\hline Média $(\Delta t)$ & $\sum \Delta t_{i} / 10=10,865 \mathrm{~s}$ \\
\hline
\end{tabular}


Calculando $T$ com os dados da Tabela 2 e aplicando-o com $L$ na equação (12), obteve-se

$$
T=\frac{\Delta t}{n}=\frac{10,865 \mathrm{~s}}{10}=1,0865 \mathrm{~s} \Rightarrow g_{2}=\frac{4 \pi^{2} \times(0,3 \mathrm{~m})}{(1,0865 \mathrm{~s})^{2}} \approx 10,0328 \mathrm{~m} / \mathrm{s}^{2}
$$

\section{$4.3 \quad$ Etapa 3}

\begin{tabular}{cc} 
Tabela 3: Dados obtidos na $3^{\mathrm{a}}$ etapa \\
\hline$m=20 \mathrm{~g}, L=0,3 \mathrm{~m}, n=15$ oscilações \\
\hline \hline Medição $(i)$ & Tempo $\left(\Delta t_{i}\right)$ \\
\hline 1 & $16,43 \mathrm{~s}$ \\
2 & $16,31 \mathrm{~s}$ \\
3 & $16,41 \mathrm{~s}$ \\
4 & $16,29 \mathrm{~s}$ \\
\hline Média $(\Delta t)$ & $\sum \Delta t_{i} / 4=16,36 \mathrm{~s}$ \\
\hline
\end{tabular}

Calculando $T$ com os dados da Tabela 2 e aplicando-o com $L$ na equação (12), obteve-se

$$
T=\frac{\Delta t}{n}=\frac{16,36 \mathrm{~s}}{15}=1,090 \mathrm{~s} \Rightarrow g_{3}=\frac{4 \pi^{2} \times(0,3 \mathrm{~m})}{(1,0907 \mathrm{~s})^{2}} \approx 9,9557 \mathrm{~m} / \mathrm{s}^{2}
$$

\subsection{Etapa 4}

Tabela 4: Dados obtidos na $4^{\mathrm{a}}$ etapa

\begin{tabular}{cc}
\hline$m=20 \mathrm{~g}, L=0,3 \mathrm{~m}, n=20$ oscilações \\
\hline \hline Medição $(i)$ & Tempo $\left(\Delta t_{i}\right)$ \\
\hline 1 & $21,90 \mathrm{~s}$ \\
2 & $21,94 \mathrm{~s}$ \\
3 & $21,97 \mathrm{~s}$ \\
4 & $22,00 \mathrm{~s}$ \\
5 & $21,88 \mathrm{~s}$ \\
\hline Média $(\Delta t)$ & $\sum \Delta t_{i} / 5=21,938 \mathrm{~s}$ \\
\hline
\end{tabular}

Calculando $T$ com os dados da Tabela 2 e aplicando-o com $L$ na equação (12), obteve-se

$$
T=\frac{\Delta t}{n}=\frac{21,938 \mathrm{~s}}{20}=1,0969 \mathrm{~s} \Rightarrow g_{4}=\frac{4 \pi^{2} \times(0,3 \mathrm{~m})}{(1,0969 \mathrm{~s})^{2}} \approx 9,8434 \mathrm{~m} / \mathrm{s}^{2}
$$

\begin{tabular}{|c|c|c|c|c|c|c|c|}
\hline \multirow{2}{*}{$\begin{array}{c}\text { Altitude } \\
\mathrm{z}(\mathrm{m})\end{array}$} & \multicolumn{7}{|c|}{ Latitude em graus } \\
\hline & 0 & 5 & 10 & 15 & 20 & 25 & 30 \\
\hline 0 & 9,7803 & 9,7807 & 9,7819 & 9,7838 & 9,7864 & 9,7896 & 9,7933 \\
\hline 50 & 9,7801 & 9,7805 & 9,7817 & 9,7836 & 9,7862 & 9,7894 & 9,7931 \\
\hline 100 & 9,7800 & 9,7804 & 9,7816 & 9,7835 & 9,7861 & 9,7892 & 9,7929 \\
\hline 150 & 9,7798 & 9,7802 & 9,7814 & 9,7833 & 9,7859 & 9,7891 & 9,7928 \\
\hline 200 & 9,7797 & 9,7801 & 9,7812 & 9,7832 & 9,7857 & 9,7889 & 9,7926 \\
\hline 250 & 9,7795 & 9,7799 & 9,7811 & 9,7830 & 9,7856 & 9,7888 & 9,7925 \\
\hline
\end{tabular}

\subsection{Cálculo dos erros}

Tabela 5: Valores da aceleração da gravidade em função da latitude e da altitude (Lopes, 2009) 
Vale ressaltar que, "devido à rotação da Terra, a aceleração gravitacional não é a mesma em todos os pontos da superfície terrestre" (Torres et al., 2016, p. 252). Por isso, para quantificar e qualificar os erros de medição, será tomado como referência o valor tabelado por Lopes (2009) de acordo com a latitude e a altitude do local. Os dados de localização (subseção 3.3) correspondem, na Tabela 5 , ao valor $g=9,7832 \mathrm{~m} / \mathrm{s}^{2}$.

Portanto, em cada etapa os erros absoluto $\left(\left|e_{i}\right|\right)$ e relativo $\left(\% e_{i}\right)$ foram:

$$
\begin{aligned}
& \left|e_{1}\right|=g_{1}-g=10,0624-9,7832=0,2792 \mathrm{~m} / \mathrm{s}^{2} \\
& \left|e_{2}\right|=g_{2}-g=10,0328-9,7832=0,2496 \mathrm{~m} / \mathrm{s}^{2} \\
& \left|e_{3}\right|=g_{3}-g=9,9557-9,7832=0,1725 \mathrm{~m} / \mathrm{s}^{2} \\
& \left|e_{4}\right|=g_{4}-g=9,8434-9,7832=0,0602 \mathrm{~m} / \mathrm{s}^{2}
\end{aligned}
$$

$$
\begin{aligned}
& e_{1}=\frac{\left|e_{1}\right|}{g} \approx 0,0285=2,85 \% \\
& e_{2}=\frac{\left|e_{2}\right|}{g} \approx 0,0255=2,55 \% \\
& e_{3}=\frac{\left|e_{3}\right|}{g} \approx 0,0176=1,76 \% \\
& e_{4}=\frac{\left|e_{4}\right|}{g} \approx 0,0062=0,62 \%
\end{aligned}
$$

\section{Conclusão}

O presente experimento mostrou que, no ensino da física, é possível aplicar materiais simples e de fácil acesso nas salas de aula do nível médio, contribuindo para um melhor e mais proveitoso aprendizado. Além disso, foram validados os valores e as equações disponibilizados pela literatura.

Como demonstrado na subseção 4.5, o valor da aceleração da gravidade obtido com o experimento se aproximou consideravelmente do valor $9,7832 \mathrm{~m} / \mathrm{s}^{2}$, tabelado por Lopes (2009) para o local do experimento. Verifica-se também uma redução considerável no erro em cada etapa. Isso se deve principalmente ao aumento do número de oscilações por medição, o que fez aumentar o espaço amostral e minimizou desvios de medição, causados sobretudo pela imprecisão humana na observação e no tempo de resposta a estímulos visuais, que influem no acionamento e na parada do cronômetro. Mesmo que o erro se reduza ao aumentar o número $n$ de oscilações, não se pode elevar $n$ exageradamente, pois o movimento do pêndulo passaria a ser amortecido, o que ocasionaria um erro ainda maior.

\section{Referências}

Doca, R. H., Biscuola, G. J., Villas, N., Bôas (2013). Física I, vol 1, $2^{\circ}$ edn. Saraiva, São Paulo.

Fuke, L. F., Yamamoto, K. (2010). Física para o Ensino Médio, vol 1, $1^{\circ}$ edn. Saraiva, São Paulo.

Halliday, D., Resnick, R., Walker, J. (2009). Fundamentos de Física, vol 2, $8^{\circ}$ edn. LTC, Rio de Janeiro.

Halliday, D., Resnick, R., Krane, K. S. (2013). Fúsica, vol 2, 5º edn. LTC, Rio de Janeiro.

Hewitt, P. G. (2011). Física Conceitual, $11^{\circ}$ edn. Bookman, Porto Alegre.

Lopes, W. (2009). Variação da aceleração da gravidade com a latitude e altitude. Caderno Brasileiro de Ensino de Física, 25(3), 561-568, URL https://periodicos.ufsc.br/index.php/fisica/article/view/2175-7941.2008v25n3p561.

Werner da Rosa, C., Becker da Rosa, A. (2012). O ensino de ciências (física) no brasil: da história às novas orientações educacionais. Revista Ibero-americana de Educação, 58(2), 1-24, URL https://rieoei.org/RIE/article/view/1446.

Torres, C. M. A., Ferraro, N. G., de Toledo Soares, P. A., Penteado, P. C. M. (2016). Física, vol 1, $4^{\circ}$ edn. Moderna, São Paulo.

Young, H. D., Freedman, R. A. (2008). Física II, $12^{\circ}$ edn. Addison Wesley, São Paulo. 